\title{
Portugal und Afrika: Die neokolonialistische Unmöglichkeit
}

\author{
Von Pedro Assunção
}

Mit der 1974er 'Revolution der Nelken` ging für Portugal eine jahrhundertlange Imperialgeschichte zu Ende. Das korporatistische Regime, das seit 1926 das politische und wirtschaftliche Geschehen in Portugal und seinen Kolonien diktierte, löste sich auf, und es kamen neue gesellschaftliche und politische Kräfte zur Geltung, die eine neue Definition der Rolle Portugals in der Welt und eine Neuorientierung der Entwicklung Portugals verlangten. Diese Entwicklung hatte ihre Begründung in den zurückliegenden Jahren, in denen sich eine Umorientierung der portugiesischen Wirtschaft und Gesellschaft auf die europäischen Länder abzeichnete. Die Kolonien, einst Prüfstein einer nationalistischen Politik wurden mehr und mehr als Last empfunden, zumal Krieg in Angola, Mosambik und Guinea-Bissau herrschte, ohne absehbaren Ausgang.

Eine Änderung der kolonialistisch-imperialistischen Bindung in Richtung auf eine neokolonialistische Auflösung des Imperiums war schon zu Anfang der 60er Jahre (mit dem organisierten militärischen Widerstand in den Kolonien) zu einer Notwendigkeit geworden, mit der sich die portugiesischen herrschenden Klassen aber schwer taten.

\section{Verhältnis zu den Kolonien bis $\mathbf{1 9 6 0}$}

Während alle kolonialen Mächte ihre Kolonien in die Unabhängigkeit zu entlassen gedachten, beharrte Portugal auf dem imperialen Traum und versuchte, die Kolonien noch strenger an die Metropole zu binden. Der II. Weltkrieg bot eine gute Möglichkeit dafür, die Kolonien gegen ausländische Interessen abzuschotten und die kolonialen Bindungen an das Mutterland zu festigen. Portugal war im II. Weltkrieg neutral geblieben, und das konnte diesem Vorhaben von Nutzen sein (Clarence-Smith, 1985a:31 f.). Dies paßte ganz in die Linie, die schon vor dem II. Weltkrieg der 'Estado Novor verfocht: Die Kolonien sollten für die alleinige Ausbeutung durch Portugal da sein und nicht ausländischen Interessen dienen (Smith, 1974:666).

Die autonome Kolonialpolitik Portugals bis Anfang der 60er Jahre basierte auf dem (1930 eingeführten) 'Acto Colonial', der 'Codigo do Trabalho Indigena' (1928) und natürlich der Verfassung von 1933 (Newitt, 1981:185). Dieser gesetzliche Rahmen zielte darauf hin, die portugiesische Einheit sowohl politisch als auch ökonomisch zu behaupten. Politisch bewirkten sie einen zentralen Aufbau des Staatsapparates in Bezug auf das ganze Imperium, also eine verringerte Autonomie für die Kolonien und einen sehr kleinen Handlungsspielraum (Smith, 1974:665). Wie Newitt (1981) zusammenfaßt: 
The post of high commissioner was abolished, and legislative and financial power was concentrated in the hands of the minister of the colonies. The minister was given the power to legislate for the colonies by decree, was to be responsible for all major appointments and for ratifying concessions. He alone could authorize loans and through government economic and commercial agencies he took an increasingly active part in the internal affairs of the territories. The governor-generals were reduced to the role of being heads of the executive, with very little initiative of their own in policy making (1981:186).

Ókonomisch brachte der 'Estado Novor und sein brillantester Kopf (Oliveira Salazar) eine protektionistische und gar merkantilistische Politik für Portugal und die von ihm beherrschten Gebiete gegenüber der Außenwelt. Das Ziel der Politik des 'Estado Novor war die Integration der Wirtschaft der Metropole mit denjenigen der Kolonien, insbesondere von Angola und Mosambik. Diese Philosophie basierte auf der Komplementarität der Produktionsstrukturen. Danach sollten die Kolonien die Metropole mit billigen Rohstoffen beliefern und diese ihrerseits sollte die verarbeiteten Produkte an die Kolonien liefern. Geschehen konnte dies aber nur unter strengen bürokratischen Kontrollen, da die Qualität und Wettbewerbsfähigkeit portugiesischer Produkte im Vergleich zu denen seiner europäischen und nordamerikanischen Partner einiges zu wünschen übrig ließ. Das autark-koloniale Modell (Esser, 1981:114) war aber nicht darauf gerichtet, die Entwicklung der Kolonien und ihrer Rohstoffvorkommen eindeutig voranzutreiben. Angestrebt wurde eher die Stabilität, ohne die das korporatistische Regime des 'Estado Novor nicht überleben konnte (Smith, 1974:663), und dies galt auch für die Kolonialpolitik. Aufgrund der Schwächen der inneren Wirtschaft mußte die Abhängigkeit auf politischem Gebiet erreicht werden. In diesem Prozeß der "Renationalisierung" der Kolonien wurden verschiedene Maßnahmen ergriffen, die das Festhalten Portugals an seinen kolonialen Gebieten und eine assimilationistische Politik unterstreichen sollten.

Bezeichnend für die autonome Kolonialpolitik Portugals in dieser Epoche war der sprunghafte Anstieg des Handels der Metropole mit den Kolonien. Hohe Handelsbarrieren wurden für ausländische konkurrierende Produkte aufgebaut (Mittelman, 1981:29 f.).

Mit dem schnellen Aufholen der europäischen Wirtschaften, die im Gegensatz zu Portugal am Zweiten Weltkrieg teilgenommen hatten, blieben die Kolonien ein unentbehrlicher Markt für Waren, die nicht mehr woanders absetzbar waren (Grupo Pesquisa, 1979:120). Portugals Außenhandelsstatistik spiegelt dies wider: während im Jahre 1938 die Kolonien $12 \%$ der portugiesischen Produkte abnahmen, stieg dieser Anteil 1948 auf $28,5 \%$.

Eigentliches Ziel dieser Politik war es, die Eigenversorgung des portugiesischen Herrschaftsbereichs in einigen Produkten gemäß der vorher beschriebenen (und wohl typischen) Arbeitsteilung zu erreichen (Newitt, 1981:191). Diese Produkte waren zunächst (hauptsächlich) Baumwolle und Zucker.

Das Beispiel Baumwolle ist in dieser Hinsicht typisch: Bei einem Konsum von $1700 \mathrm{t}$ bezog Portugal 1925 nur $800 \mathrm{t}$ aus seinen Kolonien, also $5 \%$. Im Jahre 1938 war aber diese Quote bereits auf $25 \%$ gestiegen (nachdem 1926 der Zwangsanbau durch die Ein- 
heimischen eingeführt und 1932 ein Mindestpreis garantiert wurde), 1942 lag sie bei $90 \%$ und erreichte $195095 \%$, wobei Portugal die ganze Produktion abnahm (ausgenommen den Konsum für lokale Bedürfnisse der Anbauer) (Clarence-Smith, 1985a:313; Newitt, 1981:192, 121).

Bei der Zuckerproduktion kann ein ähnlicher Prozeß beobachtet werden. Da dieses Anbauprodukt in Mosambik hauptsächlich von ausländischen Interessen bestimmt war (Sena Sugar Company), wurden durch eine Quotenaufteilung des portugiesischen Marktes die den Portugiesen gehörenden Plantagen in Angola bevorzugt. Diese konnten die Produktion ausdehnen, da sie wegen hoher Zölle auf ausländischen Zucker unter dem Verkaufspreis produzieren konnten. Um 1930 lag Portugals Konsum von Zucker bei $70000 \mathrm{t}$, wovon (theoretisch) $50 \%$ Angola und $50 \%$ Mosambik zustanden, obgleich Mosambik $70744 \mathrm{t}$ und Angola spärliche $15579 \mathrm{t}$ produzierten (Vail and White, 1980:258 f.; Newitt, 1981:192 f.). Der springende Punkt war eben, daß

Mosambik was sugar, and sugar was Hornung, Hornung was foreign, and thus it was the Angola sugar industry that was serving the true interests of Portugal (Vail and White, 1980:261).

Wie diese zwei landwirtschaftlichen Produkte wurden auch andere tropische Erzeugnisse in ähnlichen Quoten und Mindestpreissystemen reguliert (Newitt, 1981:193).

Im Industriesektor unterlagen die Kolonien seit 1936 dem 'condicionamento industriak (in der Metropole schon in Kraft seit 1931), wonach keine Produzenten zugelassen wurden, wenn die schon niedergelassenen Betriebe über genug Kapazität verfügten bzw. ihre Produktionskapazitäten nicht ausgelastet waren (Baklanoff, 1978:107 f.; Ferreira, 1985:87). Indem die Kolonien einen erweiterten Markt für die Metropole darstellten, waren die Konsequenzen dieser Politik verhängnisvoll für die Entwicklung der verarbeitenden Industrie in den kolonialen Gebieten.

Erst in den 60er Jahren wurde diese Politik aufgehoben, mit den Reformen von 1961, die 1965 zu dem 'condicionamento macional führten (Ferreira, 1985:98), die den Aufbau von Industrien in den Kolonien erlaubte, auch wenn genügend produktive Kapazität in der Metropole vorhanden war.

Trotz dieses oligopolfördernden Systems konnten sich einige industrielle Kräfte durchsetzen und industrielle Betriebe in den Kolonien (hauptsächlich Angola und Mosambik) aufbauen. In der Textilindustrie beispielsweise konnte 1944 die erste und einzig erlaubte Fabrik ihre Produktion in Angola aufnehmen, während in Mosambik die Erlaubnis für die Inbetriebnahme einer weiteren gegeben wurde (Clarence-Smith, 1985a:318; Newitt, 1981:192). Aber die protektionistische Politik der Metropole führte dazu, daß bis 1965 auf diesen zwei Territorien keine weiteren Fabriken in dieser Branche erlaubt wurden (Abshire, 1969:305). Dies zeigt auch wie, sogar bei den starken Lieferungsengpässen, die in den Kolonien durch den II. Weltkrieg entstanden, die lokale Bourgeoisie (eher um Handel und Plantagenwirtschaft besorgt) nicht imstande war, eine eigene Industrie aufzubauen, wenn dies mit Wirtschaftsinteressen in der Metropole zusammenstieß. Obwohl das Salazar-Regime eine klare nationalistische Wirtschaftspolitik betrieb, war es an der Entwicklung der Kolonien, und somit an der Ausdehnung der Märkte für portugiesische 
Produkte, nicht sehr interessiert (Grupo Pesquisa, 1979:126). Erst unter dem Druck der Politiker des 'Estado Novo' konnten die Industriellen in der Metropole dazu bewegt werden, in die Kolonien zu investieren, um einfache industrielle Einheiten, in Branchen wie Seifen-, Fisch- oder Zuckerverarbeitungsindustrie, aufzubauen. Sicherlich war es einfacher, sich auf näherliegende Märkte, wie die europäischen, zu konzentrieren, was den weiteren Vorteil eines wesentlich besser ausgebauten Transportwesens bedeutete. Außerdem steckte die portugiesische Industrie Ende des II. Weltkrieges noch in den Kinderschuhen; die weitere Entfaltung litt unter dem Mangel einer entwicklungsbewußten Bourgeoisie. 1950 war immer noch die Hälfte der portugiesischen Arbeitskräfte in der Landwirtschaft und nur 1/4 in der Industrie tätig (Baklanoff, 1978:124).

In den 50er Jahren kam es dann zu den ersten Entwicklungsplänen sowohl für die Kolonien als auch für die Metropole. Die ersten Pläne (1953-1958 und 1959-1964) stellten wenige Mittel zur Verfügung (für Angola 2,9 bzw. 4,6 Mrd. Esc.), die sich noch dadurch verringerten, daß sie nur zu 50 \% vollzogen wurden (Newitt, 1981:237). Für die Úberseeprovinzen sahen diese ersten Pläne hauptsächlich Investitionstätigkeiten im Infrastruktur- und Bewässerungsbereich vor (Grupo Pesquisa, 1979:126). Für Bereiche wie Ausbildung oder Sozialdienste sahen sie gar keine (wie im Falle des ersten Planes) oder nur wenige Ausgaben vor (im zweiten Plan $6 \%$ der Ausgaben in Angola und $14 \%$ in Mosambik für Bildung und Gesundheit - Clarence-Smith, 1985b:166). Die Erziehungsaufgaben wurden der katholischen Kirche überlassen, die die christianisierende Mission der afrikanischen Völker übernahm, einer der ideologischen Hauptpfeiler der portugiesischen Uberseeexpansion. In diesen Entwicklungsplänen ging es hauptsächlich darum, die Ausbeutung der mineralischen Rohstoffe und der Agrarprodukte zu ermöglichen. Damit war auch die Möglichkeit gegeben, die Kolonien für das portugiesische Kapital und für potentielle Siedler interessant zu machen. Die Finanzierung dieser Pläne stützte sich vorwiegend auf nationale Ressourcen, wobei in Angola und Mosambik die Finanzierungsquellen größtenteils intern zu finden waren (58 \% bzw. $95 \%$ für den ersten Plan - Brandenburg, 1969:226). Außerdem gab die Metropole keine Zuschüsse, sondern lieh das Geld unter normalen Marktbedingungen aus. Darin bestand eines der Hauptmerkmale der Finanzpolitik des 'Estado Novo', nämlich ein ausgeglichenes Budget gerade in den Kolonien zu erstreben. Der Spielraum für die Entwicklung der kolonialen Gebiete war daher sehr begrenzt, da diesen auch nicht erlaubt war, auf den internationalen Finanzmärkten Anleihen zu tätigen.

\section{Die Kolonialpolitik in den 60er Jahren}

Die durch die Politik des 'Estado Novo betriebene Isolation des portugiesischen Machtbereichs kam Ende der 50er Jahre zu einem Halt. Das Abbröckeln der anderen Kolonialreiche und die dadurch erlangte politische Unabhängigkeit der meisten kolonisierten Gebiete Afrikas und Asiens vermochten zwar die offizielle Politik Portugals gegenüber seinen Ubberseegebieten nicht zu ändern, veranlaßten jedoch die herrschenden Klassen in 
der Metropole zu einer veränderten Aufmerksamkeit in den kolonialen Gebieten. Was anderen schon längst mißlungen war, fiel erst jetzt den in Portugal bestimmenden Gruppen ein: die Integration und Assimilation der afrikanischen Kolonien und ihrer Völker wurde groß geschrieben. Anstelle der Einteilung der Einheimischen in Zivilisierte und Unzivilisierte ('indigenas', 'assimilados`), die die Gesellschaft in den Úberseeprovinzen prägte, wurden ihre Einwohner zu portugiesischen Staatsbürgern erklärt (Bailey, 1969:165). Dieses Dekret (Nr. 43893) in Verbindung mit anderen Gesetzen, die für das Leben der Einheimischen einige Anderungen in bezug auf Landbesitz, Verwaltungsorganisation und individuellen rechtlichen Status brachten, waren im Grunde die Antwort der kolonialen Instanzen auf das Aufkommen der Befreiungsbewegungen und die internationale Verurteilung der bisher betriebenen Kolonialpolitik Portugals (insbesondere im Rahmen der OAU und UNO) (Bender, 1978: 108 f., 155). Mehr denn je war die Position Portugals in Afrika von der wirtschaftlichen und militärischen Unterstützung sowohl der NATO-Länder als auch von den regionalen Nachbarn im südlichen Afrika (Südafrika und Rhodesien) abhängig. Die Offnung nach dem Westen und die darauffolgende zunehmende Vernetzung der portugiesischen Wirtschaft in Westeuropa ist die Krönung dieses Prozesses, den der Ausbruch des Befreiungskrieges in den Kolonien (1961 in Angola, 1963 in Guinea-Bissau und 1964 in Mosambik) nur noch beschleunigen sollte (Newitt, 1981:221).

Für die Weiterexistenz des portugiesischen Imperiums war die Industrialisierung der Kolonien notwendig. Nur sie konnte im Hinblick auf die Integration der Wirtschaftsräume der Metropole und der Úberseegebiete auf längere Sicht garantieren, daß das Imperium weiterbestehen würde. Die Komplementarität dieser Industrialisierung mit der der Metropole stellte sich aber rasch als trügerisch heraus, da die Ókonomien der Kolonien (Angola und Mosambik) zunehmend im Wettbewerb mit der Metropole standen (außer bei den tropischen Produkten). Die Industrialisierung und Erschließung von Rohstoffquellen machte weiterhin die Bereitstellung großer finanzieller Mittel notwendig. Hinzu kam das Versagen der bis dahin betriebenen Bildungspolitik und der große Mangel an ausgebildeten Arbeitskräften (sei es auch nur in Bezug auf die Fähigkeit, lesen und schreiben zu können).

Es waren sicherlich die Befreiungsbewegungen, die den portugiesischen Status quo am weitgehendsten unter Druck gesetzt haben. Trotz der Uberraschung, die die Revolten in Angola (zwischen November 1960 und März 1961) für die portugiesischen Machthaber darstellten, reagierten sie prompt mit dem Aufbau einer Kolonialarmee, die schon seit 1964 an drei Fronten zu kämpfen hatte. Im Jahre 1968 war die Truppenstärke schon auf mehr als 200000 (60 000 im Jahre 1960) angewachsen, ohne daß ein eindeutiger Sieg über die Befreiungsbewegungen in Sicht war (Porch, 1977:38). Die militärischen Ausgaben stiegen dann sprunghaft. Ab 1967 und bis 1970 stabilisierte sich der Anteil der militärischen Ausgaben im portugiesischen Haushalt auf 44 \% (Ferreira, 1975a:42). Das war ohne Zweifel ein Ressourcen verschlingender Krieg, der sich auf die Wirtschaft zwar nachfragesteigernd auswirkte, der aber natürlich wenig Spielraum für entwicklungsbedürftige Sektoren übrigließ. Diese starke militärische Antwort hat nicht nur einen öko- 
nomischen Hintergrund. Eine Dekolonisierung konnte sich den herrschenden Klassen nicht als Lösung zeigen, da die schwache wirtschaftliche Kontrolle dann nicht mehr haltbar wäre. Deswegen hatte ja auch das Regime ausländischen Investoren bis dahin Mißtrauen entgegengebracht.

Aber jetzt brauchte Portugal Rückenstärkung von den westlichen Industrieländern, mit denen es sich sowieso schon längst zusammengetan hatte, nämlich 1949 mit der Unterzeichnung des NATO-Vertrags. Von seinen Vertragspartnern bekam Portugal während des Kolonialkrieges militärische Hilfe und Material, obwohl der NATO-Pakt sich im Grunde nur auf den nordatlantischen Raum beschränkte (s. Minter, 1972:100-113; Maxwell, 1982 und 1985).

Der andere wichtige Partner war die Republik Südafrika. Ihre ökonomischen Interessen sowohl in Angola (e.g. das Cunene Projekt) als auch in Mosambik (z. B. der Cabora-Bassa-Staudamm) zeigen, in welchem Maße die Unterstützung der portugiesischen Politik im südlichen Afrika eine Notwendigkeit für sie wurde. Hinzu kam die regionale Verflechtung, die schon die Mosambik-Konvention von Anfang des XX. Jahrhunderts vertraglich regelte. Hinsichtlich des Arbeitsmarktes (Éntsendung von mosambikanischen Arbeitskräften in die südafrikanischen Minen) und als Devisenquelle für Portugal durch den Transport von südafrikanischen Gütern und die Bezahlung der Gastarbeiter in den Goldminen bildete dieser Vertrag den Rahmen der Abmachungen zwischen Portugal und Südafrika (Weimer, 1983:48-50; Meyns 1982:366).

Trotz allem wurde das internationale Drängen auf eine Einleitung der Entkolonialisierung der Úberseeprovinzen Portugals immer größer. In der UNO, wo seit Mitte der 50er Jahre eine wachsende Anzahl von Ländern aus Afrika und Asien, gerade in die Unabhängigkeit entlassen, ihren Platz einnahmen, stieß Portugal auf scharfe Kritik wegen seiner aggressiven Politik gegen die Befreiungsbewegungen und seines Festhaltens am kolonialen Modell. Um sich der Aufsicht des Treuhandrates zu entziehen, hatte Portugal (1951) seine Kolonien Provinzen genannt, wodurch sie formal-rechtlich keinen Unterschied zu der Metropole aufwiesen. Diesen Standpunkt konnte Portugal nicht durchsetzen, und zumindest seit 1965 veröffentlichten die Vereinten Nationen regelmäßig einen Bericht über die ökonomische Lage in den portugiesischen Kolonialgebieten.

Die Wende, die dann in der portugiesischen Kolonialpolitik einsetzte, ist durch diese soeben beschriebenen Faktoren bestimmt. Insbesondere war Angola und Mosambik angeht, war ein von Zöllen und Barrieren befreiter Warenverkehr vonnöten, um die angestrebte Integration des Imperiums zu sichern. Dies postulierte auch das Gesetz Nr. 44016 vom 18. 11. 1961, das den Traum vom multirassischen Staat und einer gemeinsamen Währung verwirklichen sollte (Graham, 1975:16). Durch das, was Perry Anderson (1962) Ultra-Kolonialismus nannte, war Portugal nicht imstande, durch eine ökonomische Kontrolle die Kolonien in die Unabhängigkeit entlassen zu können. Ferner mußte es zu protektionistischen Maßnahmen zurückgreifen, um die Ausbeutung der Kolonien zu seinen Gunsten sicherzustellen.

Seit Ende der 50er Jahre wurde aber die Offnung der Kolonien für das ausländische Kapital in Form von Direktinvestitionen und Engagement ausländischer Konzerne in den 
Kolonien, und dies nicht mehr in der klassischen Form von Konzessionsgesellschaften (hierzu Vail, 1976), wie sie seit dem XIX. Jahrhundert üblich war, zur Notwendigkeit (Boavida, 1981:115). Diese neue Politik des 'Estado Novo fand ihren Niederschlag in den Entwicklungsplänen der 60er Jahre, die eine weitgehende Auslandsfinanzierung vorsahen. Der dritte Entwicklungsplan (1968-1973) sah vor, daß $36 \%$ seiner Finanzierung vom Ausland kommen sollte. Für Angola lag dieser Anteil mit $43 \%$ noch höher. ${ }^{1}$ In allen Bereichen, insbesondere aber im Sektor der Rohstoffgewinnung, sahen sich sodann verschiedene multinationale Unternehmer durch großzügige Gesetzgebung und billige Arbeitskräfte begünstigt (Ferreira, 1975c:50 f.). Angola, eines der potentiell mineralreichsten Gebiete Afrikas, war bevorzugtes Ziel ausländischer Investoren. Zu den Bereichen, in denen ausländische Interessen schon Fuß gefaßt hatten, wie die Diamantengewinnung oder das Transportwesen (e.g. Benguela-Bahn, die für den Transport mineralischer Rohstoffe - vorwiegend Kupfer - aus Zaire und Sambia wichtig war) kam die Erdölgewinnung sowohl in Cabinda durch die Gulf Oil Company als auch in den Tiefebenen der Flüsse Congo und Kwanza durch die belgische Firma Petrofina (Castro, 1980:159 ff.; Schümer, 1977:11-20). Ferner waren ausländische Firmen in der Eisenerzgewinnung tätig, die einen beträchtlichen Impuls erhielt, nachdem die Krupp AG die zwei bestehenden Konzessionsfirmen unter ihre finanzielle Obhut nahm und diese kräftig finanzierte (Ferreira, 1975c:40-47). Das Ergebnis war, daß von 1962 bis 1971 der Export von Eisenerz um $805 \%$ anstieg und damit die vierte Stelle (10\%) unter den von Angola exportierten Produkten einnahm (s. Schümer, 1977: Tab. 89).

Höhepunkt dieser Entwicklung war das Cunene-Projekt, das an der Grenze mit Namibia ein wichtiges Instrument zur Stromversorgung wurde und durch Bewässerungsanlagen Möglichkeiten zur Einwanderung von vielen Tausenden von Weißen bieten sollte. Die erste Phase dieses großangelegten Projekts, die 1969 anlief, wurde von Südafrika vollständig finanziert (Schümer, 1977:304-311).

Genauso wie in Angola versuchten es ausländische Investoren in Mosambik. Nicht nur im Cabora-Bassa-Staudamm, der das mosambikanische Gegenstück zum Cunene Projekt darstellte, sondern auch im Bergwerksektor und der verarbeitenden Industrie wurde Südafrika Ende der 60er Jahre der wichtigste Kapitalgeber (bzw. -anleger) in Mosambik (Turok/Maxey, 1985). Einer der Hauptträger dieser Investitionspolitik war die South Africa Anglo-American Corporation, die in fast jedem Sektor der mosambikanischen Wirtschaft Fuß gefaßt hatte (Henriksen, 1983:174; Mittelman, 1981:47 f., 57 ff.).

Insgesamt flossen zwischen 1965 und $197132 \%$ der gesamten ausländischen Direktinvestitionen in die Escudozone in diese zwei Kolonialgebiete (Ferreira, 1975c:28). Diese Offnungspolitik und die Förderung der Auslandsinvestitionen zielten darauf ab, durch eine "gerechte Verteilung" des Bodens und der Rohstoffe die finanzkräftigen Industrieländer dazu zu bewegen, eine wenn auch nicht zustimmende, so zumindest doch neutrale Haltung gegenüber dem portugiesischen imperialistischen Traum einzunehmen (Lay, 1981:50 ff.). 
Dieser Prozeß, der im einzelnen in den Kolonien beschrieben wurde, fand auch in der Metropole statt. Die während der 50er Jahre in Gang gesetzte Industrialisierung schuf nicht nur Nachfrage nach kolonialen Rohstoffen. Vielmehr benötigte die Metropole Märkte für ihre Produkte einerseits, Technologie und Kapital andererseits, über die sie nicht verfügte. Hauptsächlich ab 1965 floß ausländisches Kapital nach Portugal, nicht nur in Form von Krediten, sondern auch als Direktinvestitionen (also Beteiligung und Ankauf von Gesellschaften), nachdem die Genehmigungspflicht für Investitionen gelokkert wurde. Portugal bot in der Tat in den 60er Jahren und bis zum Beitritt Großbritanniens in die EWG sehr gute Bedingungen für die Ansiedlung ausländischer Unternehmen: die Löhne waren besonders niedrig und durch die korporatistisch organisierten Gewerkschaften stark kontrolliert; völlig freie Kapitalbewegung für Profit- oder Kapitaltransfers; Anreize durch fiskalische Isentionen sowie leichter Zugang zu günstigem internen Kredit; die Möglichkeit des vorteilhaften Zugangs zu den EFTA- und Kolonialmärkten (Santos, 1977:69; Eßer et al., 1977:38).

Das ausländische Kapital brachte andererseits wichtige Impulse für die heimische Wirtschaft, obgleich diese nur in einigen wenigen Sektoren zu entdecken waren (verarbeitende Industrie). Insgesamt betrug es 4 \% der Bruttoinvestitionen von 1964 bis 1973 (Rodrigues, 1977:22 f.). ${ }^{2}$

Die Unterzeichnung des Stockholmer-Vertrages zur EFTA-Gründung (European Free Trade Association) im Jahre 1960 wurde daher schon zur Notwendigkeit, wenn Portugal den Zugang zum Markt seines wichtigsten Abnehmers (nämlich Großbritannien mit $14 \%$ ) nicht erschwert sehen wollte (Porto 1984:87). Portugal konnte mit diesem Vertrag einerseits europäische Märkte für seine traditionellen Exporte (Kork-, Textilprodukte) sichern und andererseits seine EFTA-Partner dazu bewegen, portugiesischen Industrien einen weitgehenden, obwohl zeitlich begrenzten Schutz zu gewähren (s. EFTA, 1982:G/ 1-G/3). Dadurch erhöhte sich die Konzentration der portugiesischen Exporte noch mehr auf Großbritannien: von 13,6 \% im Jahre 1960 stieg Großbritanniens Anteil an den portugiesischen Exporten 1973, dem Jahr, in dem Großbritannien und Dänemark in die EG eintraten, auf 23,8 \%. Der EFTA-Beitritt führte auf Seiten Portugals zu einem kräftigen Zuwachs der Exporte: von 1959 bis 1973 wuchsen die Exporte jährlich um $13 \%$ gegenüber $5 \%$ von 1950 bis 1959. Der Abschluß eines Freihandelsabkommens mit der EWG 1973 belegte erneut, wie wichtig die europäischen Märkte für die portugiesische Wirtschaft geworden waren (s. Guinee 1974). Portugal legte Wert auf eine forcierte exportorientierte Industrialisierung, die von Seiten der Wirtschaften der Kolonien nicht unterstützt werden konnte (s. Rocha, 1981).

Trotz der kräftigen Bemühungen zur Einwanderung in die Kolonien von Seiten des Regimes hielt sich diese in Grenzen. Nicht nur war der Markt in den einzelnen Provinzen zu klein, sondern dort fanden auch Ansätze einer Importsubstitution in mehreren Bereichen statt. Trotzdem konnten die Kolonien ihren Anteil an den portugiesischen Exporten hal-

2 Zahlen wie Blakanoff (1978:137) sie angibt, sind schlichtweg übertrieben (27\% in 1970). 
ten, bis Ende der 60er Jahre deren Wachstum absorbieren und weiterhin ein Viertel der exportierten Güter aus Portugal kaufen.

Der institutionelle Rahmen jedoch wurde gesprengt, als in den Kolonien die Zahlungsmittel unzureichend vorhanden waren, um die Importe aus Portugal zu bezahlen. Die Folge waren Importrestriktionen und fehlende Zahlungsmittel für die Abwicklung des Handels zwischen Portugal und den Kolonien (s. Rocha, 1977). 1973 konnten die Kolonien nur noch $15 \%$ der portugiesischen Exporte abnehmen. Die Integration mit den Kolonien in einer gemeinsamen Freihandelszone mit einer Währung war zwar ein Wunsch, sie wurde dennoch später (Gesetz 'Decreto-Lei، 478/1971) aufgegeben, ohne daß den Machthabern ein neues Projekt eingefallen wäre (Graham, 1975:16).

\section{Die neokoloniale Perspektive}

Die untergeordnete Stellung Portugals in der internationalen Szene hinderte es nicht daran, eine eigene Kolonialpolitik zu betreiben und sogar eine neokolonialistische Lösung anzustreben. Die Entwicklung, die bis zu dem Putsch von 1974 in Portugal anhielt, zielte dahin, den Kolonien eine weitgehende politische Autonomie zu geben bei gleichzeitiger Beibehaltung der wirtschaftlichen und finanziellen Kontrolle.

Die industrielle Basis, die sich in Portugal langsam etablierte, bevorzugte die Bildung von großen industriellen und finanziellen Gruppen, die einer Konzentration des Kapitals in den Händen weniger Gruppen gleichkam. Anfang der 70er Jahre dominierten dann auch sieben finanzstarke Gruppen die portugiesische Wirtschaft (s. Santos, 1977:71 f). Ihr Engagement in den Kolonien und die Verflechtung mit dem international operierenden Kapital wurde für einige von ihnen (BNU, Espirito Santo, Champalimaud, Burnay, CUF) wichtig zur Ausdehnung ihrer Macht. Insbesondere nach der Entschärfung des sondicionamento industrialı im Jahre 1965 ließen sich verschiedene Industrien in den Kolonien nieder, vorzugsweise die, die nicht so lukrativ waren, wie die Textilindustrie, die auf die kolonialen Binnenmärkte abgestimmten und die mit den infrastrukturellen Investitionen verbundenen Industrien, z. B. Zement (Ferreira, 1975b:14).

Auch staatlicherseits wuchs das Engagement zur Entwicklung der Kolonien. Zwar wurden die Entwicklungshilfeausgaben, die letztlich die Ausgaben der Entwicklungspläne in den Kolonien wiedergeben, durch die ständig wachsenden Verteidigungsausgaben geschmälert, doch lagen sie im Vergleich zu den Ausgaben der anderen Entwicklungshilfeausschußmitglieder (DAC - Development Assistance Committee - der OECD) wesentlich höher. Besonders in den letzten Jahren vor 1974 wuchs der Anteil der öffentlichen Entwicklungshilfe (ODA) auf deutlich über $1 \%$ (1.4 zwischen 1971-1973) des BSP. ${ }^{3}$

3 Eigene Berechnungen nach Singer/Ansari (1977), Colaço (1973), und Dinwiddy (1973) auf der Basis von DAC-Daten. 
Während die öffentliche Entwicklungshilfe noch Anfang der 60er Jahre an die $100 \%$ der Mittel zur portugiesischen Entwicklungszusammenarbeit darstellte, zeichnete sich ab 1965 der Trend zu einem größeren Gewicht des privaten Kapitalzuflußes ab, was nochmals die vorher schon angedeutete Expansion des portugiesischen Kapitals in den Kolonien bestätigt. 1972/1973 betrug diese Hilfe dann nur noch $55 \%$ der gesamten Entwicklungshilfeausgaben und somit \$126 Mio. US. (Zweijahresdurchschnitt).

Welche Sektoren und Regionen wurden damit bevorzugt? Der dritte Entwicklungsplan (1968-1973) gibt hier Aufschluß über die Ziele der protugiesischen Administration für die Kolonien (und hier vorzugsweise Angola und Mosambik), vor allem in der industriellen Entfaltung und Rohstofferschließung: Das Gros dieses Planes machte die Förderung des extraktiven und des verarbeitenden Sektors aus (49\%), während für Transport und Kommunikationswesen $22 \%$ der Gesamtausgaben, die sich auf 1547.7 Mio. US $\$$ beliefen, vorgesehen waren (Brandenburg, 1969:229).

Obwohl bezweifelt werden darf, ob den hochgesteckten Zielen tatsächlich entsprochen wurde (Newitt, 1981:273 ff.), bleibt die Tatsache, daß sowohl das ökonomische Wachstum hohe Raten verzeichnete (z. B. 22 \% jährliche Wachstumsrate für die angolanische verarbeitende Industrie zwischen 1962 und 1968 - Torres, 1983:1103), als auch die Umwandlung der traditionellen Gesellschaft vorangetrieben wurde (Ausbau des Erziehungssystems, Verstädterung durch Abwanderung in die Slums der Großstädte, Eintritt in die Kolonialarmee). Unter dem Druck des von den Befreiungsbewegungen betriebenen Guerilla-Krieges blieb dem Regime nichts anderes übrig, als der Versuch, die Einheimischen auf verschiedenste Weise vom Vorteil der portugiesischen Herrschaft zu überzeugen. Dies schien den herrschenden Klassen zunehmend als ein unmögliches Unterfangen. Daher wurden die Stimmen derer immer lauter, die auf eine Ausweitung der Autonomie der Kolonien drängten. Diese kam auch mit den protektionistischen Maßnahmen (1972), die den Kolonien (vorwiegend in Angola) eine lokale Akkumulation ermöglichen und die Einfuhr von portugiesischen Produkten erschweren sollten. Durch Beibehaltung der Finanzkontrolle und Ermöglichung der lokalen Industrieentwicklung waren die Grundsteine für eine neokolonialistische Lösung gesetzt. Hierzu ist die Ausdehnung der Aktivitäten portugiesischer Banken in den kolonialen Gebieten (insbesondere in Angola) aufschlußreich (s. Torres, 1983:1109-1113).

Das Festhalten an den Kolonien brachte nicht nur Entwicklungshilfe- und Verteidigungsausgaben mit sich, sondern es war auch Nutzen damit verbunden. Besonders als Devisenbeschaffer für das chronische Handelsbilanzdefizit der Metropole haben sich die Kolonien als brauchbar erwiesen: Die positive Handelsbilanz der Kolonien mit dem Ausland und die negative mit Portugal - verbunden mit dem interterritorialen Zahlungssystem innerhalb der Escudozone - machte dies möglich (s. Castro, 1980; Rocha, 1977 und 1982). Im Handel entstanden auch andere klassische Vorteile in den kolonialen Beziehungen, die nicht den monetären Zusammenhängen entsprangen, nämlich die Preisvorteile, die die metropolitanische Industrie bei den kolonialen Rohstoffen erzielte. Hier scheint es, daß bei den Rohstoffen, die der Industrialisierung Portugals dienten (z. B. Baumwolle), kaum Preisvorteile erzielt wurden (Eßer et al., 1977:25). Nach einer Studie 
von Rocha (1977), der die angloanischen Exporte in die Metropole untersuchte (die das Gros der Ausfuhren der Kolonien ausmachte), fanden sich nur bei Kaffee erhebliche Preisunterschiede zu den anderen Abnehmern (vorwiegend USA) (1977:601). Wie bei anderen Kolonialreichen auch, galten für koloniale Rohstoffe Preisgarantien. Vorteile bestanden eher durch die Abwesenheit von Preisschwankungen bei Belieferungen, durch die Entbehrlichkeit von Rohstofflagern in Portugal, und, da die Zahlungen in Escudos erfolgten, durch die Ersparnis an Devisen. (Eßer et al., 1977:25).

Ein anderes Mittel zur Anhäufung von Devisen auf Kosten der Kolonien waren die Zahlungen Südafrikas in Gold für die mozambikanischen Arbeiter in den Gold- und Kohleminen (s. Meyns, 1982:366). So sind die hohen Goldbestände bei der Zentralbank in Lissabon zu erklären, die 1973895 t erreichten: schätzungsweise stammt ein Drittel dieser Reserven aus den Beziehungen mit den Kolonien (Marques/Romão, 1983:1719 f).

\section{Die Folgen}

Ein Ubergang von diesen klassischen Formen der kolonialen Bindung hin zu loseren Formen der Kontrolle der Kolonien durchs Mutterland konnte aufgrund der begrenzten Wirtschaftskraft Portugals nicht gelingen. Wie sich zeigte, war die Zeit 1974, nachdem das korporatistische Regime durch einen militärischen Putsch umgestürzt war, für eine neokolonialistische Lösung noch nicht reif. Ideologisch waren die neuen Machthaber in Portugal ohnehin nicht gewillt, gerade die wirtschaftlichen Interessen gesellschaftlicher Gruppen, die das alter Regime unterstützt hatten und von ihm profitierten, zu bevorzugen, und damit deren Uberleben sowohl in Portugal als auch in den Kolonien zu gewährleisten. Die Klassen, die die neokolonialistische Lösung verkörperten (aufgeklärte Industrieinteressen wie die Champalimaud Gruppe, Teile der weißen Siedler, aufgeklärter Mittelstand), standen vor einer Kräftekonstellation, die Ihnen wenig Spielraum ließ (s. Grohs et al., 1982:17 f.; Poulantzas, 1976:42 f.). Trotzdem hätte der weitere Aufenthalt der portugiesischen Siedler (über 500000 in Angola, 200000 in Mosambik) in den Kolonien die Sicherstellung einer neokolonialistischen Beziehung begünstigen können, da jene an den Schalthebeln der Wirtschaft saßen und die technischen Berufe ausübten. Nach ihrem Rückzug aus den Kolonien (Angola und Mosambik) - in Angola wegen des Bürgerkriegs, der nach der Unabhängigkeitserklärung begann; in Mosambik wegen der entschiedenen politischen Orientierung der FRELIMO (Frente de Libertaçao de Moçambique) - schrumpfte Portugal dann zu seiner eigentlichen Größe in der Weltpolitik. So konnten die weißen Siedler in Angola und Mosambik ihre vorherigen Besitzverhältnisse nicht behaupten und sind entweder nach Südafrika (z. Z. leben 600000 bis 800000 Portugiesen in der Republik Südafrika), Portugal (mindestens 500 000) oder in andere Länder ausgewandert (s. Bender, 1978:236). Die Commonwealth-Lösung, die Spinola (1974) durchzusetzen versuchte, schlug fehl.

Die Verhältnisse innerhalb Portugals, die mehr und mehr auf einen nicht-kapitalistischen Entwicklungsweg hinwiesen, riefen von Seiten der westlichen Partner immer mehr 
Abneigung hervor, so daß die portugiesische Regierung wenig Hilfe von dieser Seite zu erwarten hatte. Das Mißtrauen der NATO-Partner, insbesondere der USA, gegenüber dem politischen Kurs in Portugal schaffte auch keinen Spielraum für mögliche Commenwealth-Lösungen (s. Oldberg, 1982). Die lange Zeit bestimmenden Pendelbewegungen in der portugiesischen Politik (nämlich Europa vs. Afrika) stabilisierten sich nunmehr in Richtung Europa. Die Traumata, die die kolonialen Kriege hinterlassen hatten (10 000 Tote und 40000 invalide Soldaten und Zivilisten), neben den Folgen der Dekolonisierung, die die portugiesische Gesellschaft belasteten, führten zur Abwendung von Afrika (Grohs et al., 1982:29). In den Auseinandersetzungen innerhalb der portugiesischen Gesellschaft verloren dann die Kräfte, die eine gemäßigte Lösung für die Entkolonisierung suchten.

Im Gegensatz zu den anderen schwarzafrikanischen Ländern, die größtenteils ihre Unabhängigkeit ohne Streit mit der jeweiligen Metropole erreichen konnten, mußten in den portugiesischen Kolonien die Befreiungsbewegungen einen langen Krieg führen, der über zehn Jahre dauerte. Daraus resultierten auch ein anderes Bewußtsein und eine politische Gestalt, die in den anderen Ländern nicht entstehen konnte (s. hierzu Chabal, 1983b). So ist es auch zu verstehen, daß für die drei Befreiungsbewegungen (PAIGC Partido Africano para a Independencia da Guine e Cabo Verde -, FRELIMO, MPLA Movimento Popular de Libertação de Angola), die in den jeweiligen Ländern an die Macht gelangten, nicht nur die Frage des Nationalismus, sondern auch die des Neokolonialismus im Vordergrund standen. Hauptsächlich die Eigentumsfragen infolge von Enteignungen und die Zahlungen der vor der Unabhängigkeit eingegangenen Schulden bildeten eine Quelle von Reibereien zwischen Portugal und hauptsächlich den zwei größten Kolonien Angola und Mosambik. Besonders ärgerlich für die ehemalige Metropole waren die Enteignungen, die nur die Portugiesen betrafen (zu Angola s. Brummel, 1984). Der Exodus der portugiesischen Siedler gab den neuen Regierungen in den neuentstandenen Ländern Spielraum für weitgehende Enteignungen in allen Sektoren der Wirtschaft. Somit war der Weg geebnet für eine Neuverteilung der Ressourcen (z. B. Land) zugunsten der Einheimischen.

Andere Sektoren wie das Bankwesen wurden verstaatlicht. Damit fehlte den Portugiesen ein weiteres Instrument, das anderen ehemaligen Kolonialmächten neokolonialistische Rahmenbedingungen ermöglicht hatte (in Bezug auf Mosambik s. Mittelmann, 1981:104).

Alles in allem verlor Portugal bis Ende 1975 die tatsächliche Kontrolle über die afrikanischen Kolonien, ohne eine neokolonialistische Kontrolle bewerkstelligen oder perspektivieren zu können. 


\section{Literaturverzeichnis}

Abshire, David M. (1969). "Minerals Manufacturing, Power, and Communications." In David M. Abshire, und Michael A. Samuels (Hrsg.), Portuguese Africa: A Handbook. London: Pall Mall.

Anderson, Perry (1962) "Portugal an the End of Ultra-Colonialism." New Left Review, Nr. 15: 83-102; Nr. 16: 88-123; und Nr. 17: 85-114.

Bailey, Norman A. (1969). "Native and Labor Policy." In David M. Abshire, und Michael A. Samuels (Hrsg.), Portuguese Africa: A Handbook. London: Pall Mall.

Baklanoff. Eric N. (1978). The Economic Transformation of Spain and Portugal. New York, usw.: Praeger Publishers.

- (1979). "The Political Economy of Portugal's Old Regime: Growth and Change Preceding the 1974 Revolution." World Development 7: 799-811.

Bender, Gerald J. (1978). Angola under the Portuguese: The Myth and the Reality. London: Heinemann.

Boavida, Americo (1981). Angola: Cinco Séculos de Exploração Portuguesa. Lisboa: Edições 70.

Brandenburg, Frank (1969). "Development, Finance, and Trade." In David M. Abshire, und Michael A. Samuels (Hrsg.), Portuguese Africa: A Handbook. London: Pall Mall.

Brummel, Jürgen (1984). Angola: Development Opportunities and Policy Options in the Southern African Area of Conflict. Bonn: FES.

Castro, Armando de (1980). O Sistema Colonial Português em África. Lisboa: Editorial Caminho.

Chabal, Patrick (1983a). Amilcar Cabral: Revolutionary Leadership and People's War. Cambridge, usw.: Cambridge University Press.

- (1983b). "People's war, State Formation and Revolution in Africa: A Comparative Analysis of Mozambique, Guinea-Bissau and Angola." Journal of Commonwealth \& Comparative Politics 21 (3): 104-125.

Clarence-Smith, Gervase (1985a). "The impact of Spanish Civil War and the Second World War on Portuguese and Spanish Africa." Journal of African History 26: 309-326.

- (1985b). The Third Portuguese Empire 1825-1975. London: Manchester University Press.

Colaço, Francis H. (1973). Economic and Political Considerations and the Flow of Official Resources to Developing Countries. Paris: OECD.

Dinwiddy, Bruce (1973). "The international Development Situation." In Bruce Dinwiddy (Hrsg.), European Development Policies: the United Kingdom, Sweden, France, EEC and Multilateral Organizations. New York: Praeger.

EFT A (1982). Convention Establishing the European Free Trade Association. Geneva: EFTA.

Esser, Klaus, et al. (1977). Portugal: Industrie und Industriepolitik vor dem Beitritt zur Europäischen Gemeinschaft. Berlin: DIE.

- (1981). "Portugal: Unterentwickelte Metropole - Entwickelte Peripherie." In Peter Jüngst (Hrsg.), Portugal nach 1974: Regionale Strukturen und Prozesse. Kassel: Gesamthochschul-Bibliothek.

Ferreira, Eduardo de Sousa (1975a). A Decadência do Corporativismo. Lisboa: Sá da Costa.

- (1975b). Portugal e o Neocolonialismo. Lisboa: Sá da Costa.

- (1975c). Strukturen der Abhängigkeit: Wirtschaftsbeziehungen der Bundesrepublik Deutschland zu Angola und Mozambique. Frankfurt: Otto Lembeck.

- (1985). "A Lógica da Consolidação da Economia de Mercado em Angola, 1930-1974." Análise Social 21(1): 83-110.

Ferreira, Manuel A. Enes (1986). "Caracterização Empresarial, Estrutura e Condicionantes das Exportações Portuguesas para a República Popular de Angola (1976-1984)“ Estudos de Economia 5(3): 157-182.

Graham, Lawrence S. (1975). Portugal: The Decline and Collapse of an Authoritarian Order. Beverly Hills, und London: Sage.

Grohs, Gerhard, Eduardo Maia Cadete, und Michael Noelke (1982). Portugals Beitritt zur Europäischen Gemeinschaft. Bonn: Europa Union.

Guinee, Peter (1974). Portugal und die Europäische Gemeinschaft. Bonn.

Henriksen, Thomas H. (1983). Revolution and Counterrevolution: Mozambique's War of Independence, 1964-1974. Westport, Conn., und London: Greenwood Press.

Grupo de Pesquisa sobre a Descolonização Portuguesa (1979). A Descolonização Portuguesa: Aproximacao a um Estudo, Bd. 1. Lisboa: IDL.

Lay, Michael van (1981). Kirche im Entkolonisierungskonflikt: Eine Fallstudie zum Krieg um die Entkolonisierung Mosambiks 1964-1974. München: Kaiser; Mainz: Grünewald. 
Marques, Alfredo, und Antonio Romão (1983). "Croissance et Crise de l'Economie Portugaise (1960-1982)." Economies et Societés 17 (9-10-11): 1701-1740.

Maxwell, Kenneth (1982). "Portugal and Africa: The Last Empire." In P. Gifford, und W. R. Louis (Hrsg.), The Transfer of Power in Africa: Decolonization 1940-1960. New Haven, und London: Yale Unversity Press.

- (1985). "As Colónias Portuguesas e a sua Descolonização." Revista Critica de Ciencias Sociais, Nr. 15/16/17: 529-567.

Meyns, Peter (1982). "Mozambique". In Dieter Nohlen, und Franz Nuscheler (Hrsg.), Handbuch der Dritten Welt, 2. Aufl., Bd. 5. Frankfurt: Hoffman und Campe.

Minter, William (1972). Portuguese Africa and the West. New York: Monthly Review Press.

Mittelman, James H. (1981). Underdevelopment and the Transition to Socialism: Mozambique and Tanzania. New York, u. a.: Academic Press.

Newitt, Malyn (1981). Portugal in Africa: The Last Hundred Years. London: C. Hurst.

O'Brien, Jay (1974). "Portugal and Africa: A Dying Imperialism." Monthly Review 26 (1): 19-36.

Oldberg, Ingmar (1982). "The Portuguese Revolution of 1974-175 and the U.S. Foreign Policy." Cooperation and Conflict 17: 179-189.

Porch, Douglas (1977). The Portuguese Armed Forces and the Revolution. London: Croom Helm.

Porto, Manuel (1984). "Portugal: Twenty Years of Change." In Alan Williams (Hrsg.), Southern Europa Transformed: Political and Economic Change in Greece, Italy, Portugal and Spain. London: Harper \& Row.

Poulantzas, Nicos (1975). Die Krise der Diktaturen: Portugal, Griechenland, Spanien. Frankfurt: Suhrkamp.

Rocha, Edgar (1977). "Portugal, anos 60: Crescimento Económico acelerado e Papel das Relacões com as Colónias." Análise Social 13 (3): 593-617.

- (1981). "Especializacão e Crescimento Económico: alguns Aspectos do Cas o Português de 1960-74." Análise Social 17 (1): 293-313.

- (1982). "Colónias e Exportacão de Mão-de-Obra como Fontes de Divisas: Consideracões sobre a contribuição dos Emigrantes para o Subdesenvolvimento Económico Português." Análise Social 18 (3/4/5): 1053-1075.

Rodrigues, António (1977). "A Penetração e a Importância dos Capitais Externos na Economia Portuguesa." Economia e Socialismo, Nr. 10: 16-37.

Santos, Américo Ramos dos (1977). "Desenvolvimento Monopolista em Portugal (fase 1968-73): Estruturas fundamentais." Análise Social 13 (1): 69-95.

Schümer, Martin (1977). Die Wirtschaft Angolas 1973-1976: Ansätze einer Entwicklungsstrategie der MPLARegierung. Hamburg: Institut für Afrika-Kunde.

Singer, Hans W., und Javed A. Ansari (1977). Rich and Poor Countries. London: Allen \& Unwin.

Smith, Alan K. (1974). "António Salazar and the Reversal of Portuguese Colonial Policy." Journal of African History 15 (4): 653-667.

Spinola, António de (1974). Portugal e o Futuro. Lisboa: Arcádia.

Torres, Adelino (1983). "Pacto Colonial e Industrialização de Angola (anos 60-70)." Análise Social 19 (Nr. 77/78/79): 1101-1119.

Turok, Ben, und Kees Maxey (1985). "Southern Africa in Crisis." In Gutkind, und I. Wallerstein (Hrsg.), Political Economy of Contemporary Africa. London, u. a.: Sage.

Vail, Leroy (1976). "Mozambique's Chartered Companies: The Rule of the Feeble."Journal of African History 17 (3): $389-416$

- und Landeg White (1980). Capitalism and Colonialism in Mozambique: A Study of Quelimane District. London, u. a.: Heinemann.

Weimer, Bernhard (1983). Die Mozambiquanische Außenpolitik 1975-82: Merkmale, Probleme, Dynamik. Baden-Baden: Nomos Verlagsgesellschaft. 
It goes on to argue that this 'two spheres' approach continued after independence, despite a strong anti-slaw rhetoric at the time, but also draws attention to the fact that the Independence Constitution calls for the development of a new Melanesian jurisprudence as a basis for a different type of pluralistic legal system, aimed at creating modern, but nonetheless distinctly Melanesian forms of political organisation.

In other words, the constitution-makers in Papua New Guinea saw the main task of improving the relations between law' and 'custom' not as an integration of the latter into the former but in a fundamental, and primarily constitutional, reform of the former; in short: law reform rather than a recognition of custom.

\section{Portugal And Africa: The Impossibility of Neo-Colonialism}

\section{By Pedro Assunçao}

Portugal's secular empire in Africa came to an end in the wake of the coup d'état which overthrew the corporatist dictatorship established in 1926.

The article describes the attempts by the metropolitan government before 1974 to strengthen the links with its African possessions even in the face of large-scale decolonisation after the Second World War. Portugal's insufficient economic power required massive infusion of foreign capital for investment in the colonies in order to enhance conditions for further settlement by metropolitan immigrants. This economic weakness led Portugal into regional co-operation with South Africa and, after the beginning of guerilla war in the three colonies of Guinea-Bissau, Angola and Mozambique, to substantial military aid by Portugal's NATO partners.

Confronted with a deteriorating military situation in the colonies, Portugal nevertheless pursued a policy of sweeping integration of the metropolis and the overseas dominions, aiming at an sultra-colonial, multiracial union. Military dégringolade in Africa and economic impotence eventually forced Portugal to abandon both the rule of her African possessions and any hope for the reestablishment of economically based, neo-colonialist control after the departure of the colonial administration and the vast majority of Portuguese settlers. 